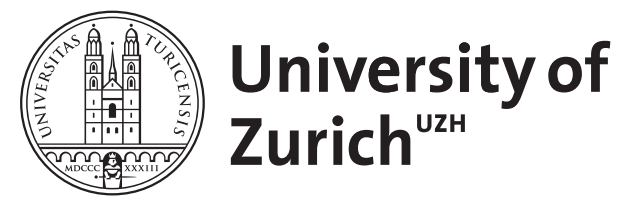

\title{
Self-induced compactness in Banach spaces
}

Casazza, P G ; Jarchow, H

\begin{abstract}
We consider the question: is every compact set in a Banach space X contained in the closed unit range of a compact (or even approximable) operator on $\mathrm{X}$ ? We give large classes of spaces where the question has an affirmative answer, but observe that it has a negative answer, in general, for approximable operators. We further construct a Banach space failing the bounded compact approximation property, though all of its duals have the metric compact approximation property
\end{abstract}

DOI: https://doi.org/10.1017/s0308210500022770

Posted at the Zurich Open Repository and Archive, University of Zurich ZORA URL: https://doi.org/10.5167/uzh-155326

Journal Article

Published Version

Originally published at:

Casazza, P G; Jarchow, H (1996). Self-induced compactness in Banach spaces. Proceedings of the Royal Society of Edinburgh Section A: Mathematics, 126(02):355-362.

DOI: https://doi.org/10.1017/s0308210500022770 


\title{
Self-induced compactness in Banach spaces
}

\author{
P. G. Casazza* \\ Department of Mathematics, University of Missouri-Columbia, Columbia, \\ Mo 65211, U.S.A. \\ e-mail: Pete@casazza.cs.missouri.edu
}

H. Jarchow $\dagger$

Mathematisches Institut, Universität Zürich, CH 8057 Zürich, Switzerland e-mail: jarchow@math.unizh.ch

(MS received 22 March 1994. Revised MS received 4 October 1994)

\begin{abstract}
We consider the question: is every compact set in a Banach space $X$ contained in the closed unit range of a compact (ot even approximable) operator on $X$ ? We give large classes of spaces where the question has an affirmative answer, but observe that it has a negative answer, in general, for approximable operators. We further construct a Banach space failing the bounded compact approximation property, though all of its duals have the metric compact approximation property.
\end{abstract}

\section{Introduction}

The question which led to the title of this paper is the following: if $X$ is a Banach space and $K$ is a compact subset of $X$, is it possible to find a compact, or even approximable, operator $v: X \rightarrow X$ such that $K \subset \overline{v\left(B_{X}\right)}$ ? This question was first posed by P. G. Dixon [6] in connection with investigating the problems of the existence of approximate identities in certain operator algebras. We shall provide some observations related to the above question and give, in particular, a negative answer in the case of approximable operators.

We shall also provide the first examples of Banach spaces having the approximation property but failing the bounded compact approximation property, though all of their duals do have even the metric compact approximation property.

\section{Approximate identities}

A left approximate identity (LAI) in a Banch algebra $A$ is a net $\left(e_{i}\right)_{i \in I}$ in $A$ such that $\lim _{i \in I}\left\|e_{i} x-x\right\|=0$ for each $x \in A$. If there is a $\lambda>0$ such that in addition $\left\|e_{i}\right\| \leqq \lambda$, then $\left(e_{i}\right)_{i \in l}$ is called a $\lambda$-bounded LAI ( $\lambda$-BLAI); note that necessarily $\lambda \geqq 1$. We say that $\left(e_{i}\right)_{i \in I}$ is a BLAI if it is a $\hat{\lambda}$-BLAI for some $\lambda$. Right approximate identities (RAl) and $(\lambda-)$ bounded $\mathrm{RAI}((\lambda-) \mathrm{BRAI})$ are defined analogously. 
A result which, when seen in the context of operator algebras, is of particular interest for Banach space theory, is P. J. Cohen's [4] factorisation theorem:

If the Banach algebra $A$ has a BLAI, then every $z \in A$ can be written as a product $z=x y$ with $x, y \in A$; in addition, $y$ exists in the closed left ideal generated by $z$ and can be chosen so that $\|z-y\| \leqq \delta$, where $\delta$ was given previously.

See F. F. Bonsall and J. Duncan [2] for details.

An interesting case occurs when $A$ is of the form $\mathscr{A}(X)$ where $\mathscr{A}$ is any Banach operator ideal in the sense of A. Pietsch [13] and $X$ is a Banach space. However, in such generality approximate identities have hardly been discussed; attention has been focused on the cases where $\mathscr{A}$ is either $\mathscr{K}$, the ideal of all compact operators, or $\overline{\mathscr{F}}$, the ideal of all approximable operators, i.e. uniform limits of operators of finite rank. Of course, $\mathscr{F}$ will be used to denote the ideal of all finite rank operators between Banach spaces. We warn the reader that our notation is the same as that of P. G. Dixon [6], but it is incompatible with that of N. Gronbrek and G. A. Willis [7], where $F$ denotes the finite rank operators and $\mathscr{F}$ the approximable operators. Dixon's paper [6] deals with precisely these ideals, and one of his main results is the following theorem:

THEOREM 2.1. A Banach space $X$ has the $\lambda$-BAP [respectively the $\lambda$-BCAP] if and only if $\overline{\mathscr{F}}(X)$ [respectively $\mathscr{K}(X)]$ has a $\lambda$-BLAI.

Recall that a Banach space $X$ has AP (approximation property) (respectively CAP (compact approximation property)) if, for any compact subset $K$ of $X$ and any $\varepsilon>0$, there is an operator $u \in \mathscr{F}(X)$ (respectively $u \in \mathscr{K}(X)$ ) such that $\|u x-x\|<\varepsilon$ for all $x \in K$. If there is a $\lambda(\geqq 1)$ such that we always can arrange for $\|u\| \leqq \lambda$, then we say that $X$ has $\lambda$-BAP (respectively $\lambda$-BCAP), with 'B' being shorthand for 'bounded', of course. Usually, BAP (respectively BCAP) is used when we only know that we have $\lambda$-BAP (respectively $\lambda$-BCAP) for some $\lambda$. The case $\lambda=1$ corresponds to what is usually called MAP (metric approxintation property) and MCAP (metric compact approximation property), respectively.

Only recently, C. Samuel [16] and N. Grønbak and G. A. Willis [7] addressed the corresponding problem of existence of RAI. One of the main results in [7] is the following companion result of the preceding theorem:

THEOREM 2.2. Let $X$ be a Banach space. Then $X^{*}$ has the $\hat{\lambda}$-BAP if and only if $\bar{F}(X)$ has a $\lambda$-BRAI.

The situation is less pleasant when $\overline{\mathscr{F}}$ is replaced by $\mathscr{K}$. It is still true that if $\mathscr{K}(X)$ has a $\lambda$-BRAI, then $X^{*}$ has $\lambda$-BCAP. However, the converse fails. As we shall see, there is a Banach space $X$ with $\mathrm{AP}$, but which fails $\mathrm{BCAP}$, while $X^{*}, X^{* *}, \ldots$ are all separable and have MCAP. If $\mathscr{K}(X)=\overline{\mathscr{F}}(X)$ has a BRAI, then $X^{*}$ would have BAP, and so $X$ should have BAP as well-but it does not. What is true here was observed by C. Samuel [16]. That is, $\mathscr{K}(X)$ has a $\lambda$-BRAI if and only if $X^{*}$ has the $\lambda$-BCAP given by $w^{*}$-continuous operators.

Our example answers a question which originates in the work of A. Grothendieck [8] and was restated as question 2 in [16]. (Question 1 in [16] has been answered by $\mathrm{G}$. A. Willis [19] and question 3 by Theorem 2.3 below.) To construct our example, we need some results from the literature. Recall that a Banach space $X$ has 
shrinking $\lambda$-CAP if there is a set $u_{\alpha} \in \mathscr{K}(X),\left\|u_{\alpha}\right\| \leqq \lambda,\left(u_{\alpha}\right)$ converges strongly to the identity on $X$ and $\left(u_{\alpha}^{*}\right)$ converges strongly to the identity on $X^{*}$. In his memoir [8], A. Grothendieck showed that a separable dual space with AP has MAP. The corresponding question for CAP is still open. However, an alternative proof of Grothendieck's result due to J. Lindenstrauss and L. Tzafriri [11] will work for shrinking CAP (see C. Cho and W. B. Johnson [3]).

THEOREM 2.3. If $X^{*}$ is separable and has CAP given by $w^{*}$-continuous operators, then $X$ and $X^{*}$ have MCAP.

It follows that:

COROLlARY 2.4. If $X^{*}$ is separable and $X$ has shrinking CAP, then for every equivalent norm $|\cdot|$ on $X$, both $X$ and $(X,|\cdot|)^{*}$ have MCAP.

We are now ready for:

THEOREM 2.5. There is a Banach space $X$ which has AP, but fails BCAP, while $X^{*}, X^{* *}, \ldots$ are all separable and have MCAP.

Proof. Let $Y$ be a separable reflexive Banach space with CAP which fails to have $A P$, see G. A. Willis [19]. Choose a Banach space $Z$ so that $Z^{* *}$ has a basis and $Z^{* *} / Z \approx Y$ (see [11, the proof of Theorem 1.e.7, p. 34]). Now, $Z^{* * *} \approx Z^{*} \oplus Y^{*}$ fails AP but has shrinking CAP since (1) $Z^{*}$ has a shrinking basis, and (2) $Y$ is reflexive and has CAP. It follows that $Y$ has shrinking CAP. By a construction of T. Figiel and $W$. B. Johnson $\left[11\right.$, p. 42], for each $n$, there is an equivalent norm $\mid \cdot \ln$ on $Z^{* *}$ so that $\left(Z^{* *},|\cdot|_{n}\right)$ fails $n$-BAP. But $\left(Z^{* *},|\cdot|_{n}\right)^{*}$ still has shrinking CAP (being isomorphic to $Z^{* * *}$ ). So by Corollary 2.4 , this space and its dual have MCAP. (A similar argument shows, in fact, that all of its duals have MCAP.)

Let

$$
X=\left(\sum_{n=1}^{\infty} \oplus\left(Z^{* *},|\cdot|_{n}\right)\right)_{l_{2}} .
$$

Then $X$ has AP and fails BAP. So $X$ fails BCAP. (It is easily seen that $X$ has $\lambda$-BAP if and only if $X$ has AP and $\lambda$-BCAP.) The spaces $X^{*}, X^{* *}, \ldots$ are all $l_{2}$-sums of Banach spaces having MCAP, and hence have MCAP. This completes the construction.

\section{The properties $(\overline{\mathscr{F}})$ and $(\mathscr{K})$}

We shall now concentrate on left-approximate units. There is a natural question related to Theorem 2.1: if the same $\lambda$ works for the BLAI and for the B(C)AP, could it not be simultaneously 'eliminated' on both sides? More precisely, is it true that $\overline{\mathscr{F}}(X)$, respectively $\mathscr{K}(X)$, has a LAI if and only if $X$ has AP, respectively CAP?

Let $\mathscr{A}$ be $\overline{\mathscr{F}}$ or $\mathscr{K}$. We say that a Banach space $X$ has the property $(\mathscr{A})$ if, for each compact subset $K$ of $X$, there is an operator $u \in \mathscr{A}(X)$ such that $K \subset \overline{u\left(B_{X}\right)}$. This is what 'self-induced compactness' in the title is referring to. Of course, the concept can be generalised in many directions, but we prefer to stay with the present setup.

The following results are again due to Dixon [6]: 
THEOREM 3.1. Let $X$ be a Banach space.

(a) If $X$ has AP (CAP), then $\overline{\bar{F}}(X)(\mathscr{K}(X))$ has a LAI.

(b) If $X$ has property $(\overline{\mathscr{F}})((\mathscr{K}))$ and if $\overline{\mathscr{F}}(X)(\mathscr{K}(X))$ has a LAI, then $X$ has AP (CAP).

(c) If $X$ has BAP (BCAP), then it has property $(\overline{\mathscr{F}})((\mathscr{K}))$.

All we need to get started is a workable condition which is equivalent to property $(\mathscr{A})$. This is elementary:

Proposition 3.2. The following statements about the Banach space $X$ are equivalent:

(i) $X$ has property $(\infty)$;

(ii) given $u \in \mathscr{K}\left(l_{1}, X\right)$, there are operators $v \in \mathscr{N}(X)$ and $w_{n} \in \mathscr{L}\left(l_{1}, X\right)(n \in \mathbf{N})$ such that $\lim _{n \rightarrow \infty}\left\|u-v w_{n}\right\|=0$;

(iii) same as (ii), but $w_{n} \in \mathscr{K}\left(l_{1}, X\right)$;

(iv) for each $u \in \mathscr{K}\left(l_{1}, X\right)$, there are operators $v \in \mathscr{A}(X)$ and $w \in \mathscr{L}\left(l_{1}, X^{* *}\right)$ such that $u=v^{* *} w$;

(v) same as (iv), but $w \in \mathscr{K}\left(l_{1}, X^{* *}\right)$.

Since we are working with (weakly) compact operators $v: X \rightarrow X$, we may and shall consider $v^{* *}$ as an operator $X^{* *} \rightarrow X$; accordingly we have

$$
\overline{v\left(B_{X}\right)}=v^{* *}\left(B_{X^{* *}}\right) \text {. }
$$

Proof of Proposition 3.2. (i) $\Rightarrow$ (ii) Given $u \in \mathscr{K}\left(l_{1}, X\right)$, we can find for each $n \in \mathbf{N}$ vectors $x_{k} \in B_{X}$ such that $\left\|u x_{k}-u e_{k}\right\| \leqq n^{-1}$ for all $k \in \mathbf{N}$, and then define $w_{n}: l_{1} \rightarrow X$ via $w_{n} e_{k}=x_{k}$ for each $k$.

(ii) $\Rightarrow$ (iii) Any $u \in \mathscr{K}\left(l_{1}, X\right)$ can be written as $u=u_{1} u_{2}$, where $u_{1}: l_{1} \rightarrow X$ and $u_{2}: l_{1} \rightarrow l_{1}$ are compact operators.

(iii) $\Rightarrow$ (iv) We may assume that $\left\|w_{n}\right\| \leqq 1$ for each $n$. Let $\mathscr{Z}$ be a free ultrafilter on $\mathbf{N}$ and define $w: I_{1} \rightarrow X^{* *}$ by $w(\xi)=\lim _{*} w_{n} \xi$, the limit being taken in the weak $*$ topology of $X^{* *}$. This is a bounded linear operator, and $u=v^{* *} w$.

(iv) $\Rightarrow$ (v) is obtained in the same way as (ii) $\Rightarrow$ (iii), so we are left with (v) $\Rightarrow$ (i). Let $K \subset X$ be compact. Then $K \subset \overline{\operatorname{conv}}\left\{x_{n}: n \in \mathbf{N}\right\}$ for some null sequence $\left(x_{n}\right)$ in $K$. The operator $u: l_{1} \rightarrow X$ defined by $u e_{n}:=x_{n}$ for each $n$ is compact, so there are $v \in \mathscr{A}(X)$ and $w \in \mathscr{K}\left(l_{1}, X^{* *}\right)$ such that $u=v^{* *} w$. We may assume that $\|w\| \leqq 1$, so that $K \subset \overline{u\left(B_{l_{1}}\right)} \subset v^{* *}\left(B_{X^{* *}}\right)$.

Since $l_{1}$ enjoys the lifting property [11, p. 108], a Banach space $X$ has property $(A)$ whenever the following applies: no matter how we choose the Banach space $Y$ and the operator $u \in \mathscr{A}(Y, X)$, we can find a quotient $Q$ of $X$ along with operators $v \in \mathscr{A}(Q, X)$ and $w \in \mathscr{L}\left(Y, Q^{* *}\right)$ such that $u=v^{* *} w$. It is interesting to note that in case $S=\mathscr{K}$, the preceding proposition allows us to extend this almost to a characterisation. We have the following weak version of the Cohen factorisation theorem:

Proposition 3.3. Let $X$ be a Banach space.

(a) If $X$ has property ( $\mathscr{H})$ then, given any Banach space $Y$, every operator $u \in \mathscr{K}(Y, X)$ admits a compact factorisation through some quotient $Q$ of $X^{* *}$ : there are operators $v \in \mathscr{K}(Q, X)$ and $w \in \mathscr{K}(Y, Q)$ such that $u=v w$.

(b) Suppose there is, for every Banach space $Y$ and every $u \in \mathscr{K}(Y, X)$, a quotient space $Q$ of $X$ together with operators $v \in \mathscr{K}(Q, X)$ and $w \in \mathscr{K}\left(Y, Q^{* *}\right)$ such that $u=$ $v^{* *} w$. Then $X$ has property $(\mathscr{K})$. 
Proof. (a) Suppose that $X$ has $(\mathscr{K})$ and let $Y$ and $u \in \mathscr{K}(Y, X)$ be given. Of course we may assume that $Y$ is separable; so we can work with a quotient map $q: l_{1} \rightarrow Y$. Thanks to Proposition 3.2 there are operators $v_{0} \in \mathscr{K}(X)$ and $w_{0} \in \mathscr{K}\left(l_{1}, X^{* *}\right)$ such that $u q=v_{0}^{* *} w_{0}$. Set $Q=X^{* *} / \operatorname{ker}\left(v_{0}^{* *}\right)$, let $p: X^{* *} \rightarrow Q$ be the quotient map, and let $v \in \mathscr{K}(Q, X)$ be such that $v p=v_{0}^{* *}$. As $v$ is injective, there is a $w \in \mathscr{K}(Y, Q)$ such that $w q=p w_{0}$. Note that $u=w v$.

(b) Apply the hypothesis to any $\mathscr{K}\left(l_{1}, X\right)$ and use the lifting property of $l_{1}$.

We do not have a corresponding result for the property $(\overline{\bar{F}})$.

We continue by giving a number of immediate consequences of Proposition 3.2. If the Banach space $X$ admits a quotient which is isomorphic to $l_{1}$ then, by the lifting property of $l_{1}$, this quotient is isomorphic to a complemented subspace of $X$. So we may state:

CoRollary 3.4. Any Banach space which admits a quotient isomorphic to $l_{1}$ has the property $(\overline{\bar{F}})$.

If $X$ fails CAP, then $X \oplus l_{1}$ fails CAP and has $l_{1}$ as a quotient space. It follows from Theorem 3.1 that for such a space $X$ neither $\mathscr{K}(X)$ nor $\overline{\mathscr{F}}(X)$ can have LAI. We can easily extend the list of such examples.

LEMma 3.5. Let $X, Y$ and $Z$ be Banach spaces. Suppose that $X$ is a quotient of $Y$, that $Z$ is a quotient of $X$, and that every $u \in \mathscr{K}\left(l_{1}, Y\right)$ can be written $u=v w$ where $w \in \mathscr{K}\left(l_{1}, Z\right)$ and $v \in \mathscr{A}(Z, Y)$. Then $X$ has the property $(\mathscr{A})$.

This is an immediate consequence of the fact that $l_{1}$ has the compact lifting property.

Corollary 3.6. Let $X$ be a subspace of $c_{0}$ or $l_{p}(1 \leqq p<\infty)$. Then $X^{*}$ has property $(\overline{\mathscr{F}})$.

But $X^{*}$ may well fail to have CAP, in which case neither $\overline{\mathscr{F}}(X)$ nor $\mathscr{K}(X)$ can have a LAI.

The proof is immediate from the lemma and the fact that every infinite dimensional subspace of $c_{0}$ or $l_{p}$ contains a subspace which is isomorphic to $c_{0}$, respectively $l_{p}$, and complemented in the whole space [11, Proposition 2.a.2, p. 53].

We do not know if every subspace of $c_{0}$ or $l_{p}(1 \leqq p<\infty)$ has $(\mathscr{K})$ or $(\overline{\mathscr{F}})$. Also, we do not know how $(\infty)$ behaves with respect to duality.

W. B. Johnson has proved in [10] that there is a separable Banach space, $C_{1}$, such that if $Z$ is any separable Banach space, then $Z^{*}$ is isometrically isomorphic to a norm-one complemented subspace of $C_{1}^{*}$. It follows that $C_{1}^{*}$ fails CAP but it has the property $(\overline{\mathscr{F}})$ since $l_{1}$ is complemented in $C_{1}^{*}$. Consequently, neither $\mathscr{K}\left(C_{1}^{*}\right)$ nor $\overline{\mathscr{F}}\left(C_{1}^{*}\right)$ can have a LAI.

On the basis of this, it is tempting to conjecture that the property $(\mathscr{A})$ is preserved under the formation of complemented subspaces. The above observation about duals of subspaces of $c_{0}$ and $l_{p}$ would then appear as a consequence of $C_{1}^{*}$ 's property of having ( $(\bar{F})$; in fact, the dual of any separable Banach space would have (F) . We shall now see that this is not the case.

Let $\Gamma_{2}$ denote the ideal of all Banach space operators which factor through some Hilbert space. 
Proposition 3.7. Suppose that the Banach space $X$ has the property $(\mathscr{A})$. If $\mathscr{A}(X) \subset \Gamma_{2}(X)$, then $X$ is isomorphic to a Hilbert space.

Proof. In fact, Grothendieck's Inequality (see [15, Proposition 6.2, p. 71]) informs us that $\mathscr{K}\left(l_{1}, X\right)$ consists of absolutely summing operators only. By trace duality, however, this can only happen when $X$ is isomorphic to a Hilbert space.

It was shown by $\mathrm{G}$. Pisier [14] that $\overline{\mathscr{F}}(X)$ is contained in $\Gamma_{2}(X)$ whenever $X$ and $X^{*}$ both have coptype 2 , and a few years later, he proved [15, Theorem 10.6, p. 71$]$ that every cotype 2 space $Z$ embeds into a non-Hilbertian space $P_{Z}$ which, together with its dual, has cotype 2 . Such a space necessarily fails AP. Thus:

Corollary 3.8. The Pisier spaces $P_{Z}$ fail to have property ( $\overline{\mathscr{F}}$ ).

The same is true for all the duals of the spaces $P_{Z}$. But thanks to Corollary 3.4, the spaces $P_{Z} \oplus l_{1}$ enjoy property $(\overline{\mathscr{F}})$. Conclusion:

COROLlaRX 3.9. The property $(\overline{\mathscr{F}})$ is not preserved when passing to complemented subspaces.

Another way to obtain this is by using Johnson's universal space $C_{1}^{*}$ : if $Z$ is separable, then Pisier's construction leads to a separable space $P_{Z}$, so that $P_{Z}^{*}$ is complemented in $C_{1}^{*}$.

Actually, the spaces $P_{Z}$ enjoy even more exotic properties. For example, $\overline{\bar{F}}\left(P_{Z}\right)$ coincides with $\mathscr{N}\left(P_{Z}\right)$, the algebra of all nuclear operators $u: P_{Z} \rightarrow P_{Z}$. We do not know if $\overline{\mathscr{F}}\left(P_{Z}\right)=\mathscr{N}\left(P_{Z}\right)$ can have a LAI; actually, the question of what the meaning of the existence of LAI in $\mathscr{N}(X)$ is in terms of $X$ does not seem to have been investigated. However, it was recently shown by Y. V. Selivanov [17] that $\mathscr{N}(X)$ has a BLAI if and only if $X$ is finite dimensional; see also $H$. G. Dales and $H$. Jarchow in [5]. The arguments employed before also show that $\Gamma_{2}\left(P_{z}\right)$ does not have a LAI.

\section{Odds and ends}

We start by listing a few open problems:

(a) Are there Banach spaces failing $(\mathscr{K})$ ?

(b) Are there Banach spaces failing $(\mathscr{K})$ such that $\mathscr{K}(X)$ has (does not have) a LAI?

(c) Can any of the algebras $\overline{\mathscr{F}}\left(P_{Z}\right)$ have a LAI?

(d) When do the algebras $\mathscr{N}(X), \Gamma_{2}(X), \ldots$ have a LAI?

(e) How do the properties $(\mathscr{K})$ and $(\overline{\overline{\mathscr{K}}})$ behave with respect to duality?

(f) Regarding $(\mathscr{K})$ and ( $(\overrightarrow{\mathscr{F}})$, what can be said when the underlying Banach space $X$ is a Banach lattice, $H^{\infty}$, a $C^{*}$-algebra, ...?

Here are some further ideas which lead to many more problems. The property $(\mathscr{A})$ can be generalised as follows. Given a Banach space $X$, let $\mathscr{A}_{X}$ be the collection of all Banach spaces $Z$ such that for each compact subset $K$ of $X$ there is an operator $u \in \mathscr{A}(Z, X)$ such that $K \subset \overline{u\left(B_{Z}\right)}$; again $\mathscr{A}$ is $\mathscr{K}$ or $\overline{\mathscr{F}}$.

It seems plausible that investigation of such a concept could be helpful in understanding compactness in general Banach spaces through known characterisations of compactness in e.g. classical spaces. Not much, however, is known, and what is known indicates that the picture will by no means be easy to understand. 
There are Banach spaces $Z$ which belong to $\cap_{X} \cdot \overline{\mathscr{F}}_{X}: l_{1}$ and the duals of Johnson's spaces $C_{p}$ provide examples.

If $X$ has BAP (respectively BCAP), then $X$ belongs to $\overline{\mathscr{F}}_{X}$ (respectively $\mathscr{K}_{X}$ ), whereas the Pisier spaces $P_{Z}$ satisfy $P_{Z} \notin \overline{\bar{F}}_{P_{Z}}$.

These spaces also satisfy $P_{Z} \notin \mathscr{K}_{\left(P_{Z^{\prime}}\right)^{*}}$. In fact, K. John [9] has shown that every compact operator $P_{Z} \rightarrow\left(P_{Z}\right)^{*}$ is nuclear, so that $P_{Z} \in \mathscr{K}_{\left(P_{Z}\right)^{*}}$ would entail $\left.\mathscr{K}\left(l_{1}, P_{Z}\right)^{*}\right)=\mathscr{N}\left(l_{1},\left(P_{Z}\right)^{*}\right)$ which cannot be reconciled with $P_{Z}$ being infinite dimensional.

On the other hand, there are Banach spaces $X$ such that $X \in \overline{\bar{F}}_{X^{*}}$ : think of $X=$ $l_{1}, X=C_{p}, X=l_{2}, \ldots$ Can one characterise such spaces?

By the same type of argument, we get that if $X$ is a non-Hilbertian cotype 2 space, then $\overline{\mathscr{F}}_{X}$ cannot contain any $Z$ such that $Z^{*}$ has cotype 2 . In fact, otherwise we would get $\mathscr{K}\left(l_{1}, X\right)=\Gamma_{2}\left(l_{1}, X\right)$ (cf. [14]), and this is only possible if $X$ is isomorphic to a Hilbert space. Similarly, if $X$ has cotype 2 and if $\mathscr{K}_{X}$ contains a Banach space of type 2 , then $X$ must be Hilbertian.

All these examples revolve around Hilbert space and amount to the conclusion that $\mathscr{K}_{X}$ contains a Hilbert space if and only if $X$ is isomorphic to Hilbert space.

We claim that, if $X$ is Hilbertian, then $\mathscr{K}_{X}$ contains even all dual Banach spaces. (All spaces are supposed to be infinite dimensional.) This can be seen as follows. Let $u: l_{1} \rightarrow X$ be a compact operator. Since $X$ is Hilbertian,

$$
u: l_{1} \stackrel{u_{2}}{\rightarrow} l_{2} \stackrel{u_{1}}{\rightarrow} X
$$

where $u_{1}$ and $u_{2}$ are compact operators. Now let $Z$ be any Banach space. By a result of S. Bellenot [1] (J. S. Morell and J. R. Retherford [12]) there exists a quotient space $Q$ of $Z^{*}$ and compact operators $v_{1}: Q \rightarrow X$ and $v_{2}: l_{2} \rightarrow Q$ such that $u_{1}=v_{1} v_{2}$. Invoke the compact lifting property of $l_{1}$ to finish the proof.

The situation resembles the one encountered in Proposition 3.3. Question: can one get a factorisation through $Z$ rather than through $Z^{*}$ ?

\section{References}

1 S. Bellenot. The Schwartz. Hilbert variety. Michigan Math. J. 22 \{1975\}, 3737.

2 F. F. Bonsall and J. Duncan. Complete Normed Algebras (Berlin: Springer, 1973).

3 C. Cho and W. B. Johnson. A characterization of subspaces $X$ and ${ }^{\prime \prime}$ for which $K(X)$ is an M-ideal in $L(X)$. Proc. Amer. Math. Soc 93 (1985), 466-70.

4 P. J. Cohen. Factorization in group algebras. Duke Math. J. 26 (1959), 199-205.

5 H. G. Dales and H. Jarchow. Continuity of homomorphisms and derivations from algebras of approximable and nuclear operators. Math. Proc. Cambridge Philos. Soc. 116 (1994), 465-73.

6 P. G. Dixon. Left approximate identities in algebras of compact operators on Banach spaces. Proc: Roy. Soc. Edinhurgh Sect. A 104 (1986), 16975.

7 N. Gronbak and G. A. Willis. Approximate identities in the Banach algebras of compact operators. Canad Math. Bull. 36 (1993), 45-53.

8 A. Grothendieck. Produits tensoriels topologies et espaces nucléaires. Mem. Amer. Math. Soc. 16 (1955).

9 K. John. On the compact non-nuclear problem. Math. Ann. 287 (1990), $509: 14$.

10 W. B. Johnson. A complementary universal conjugate Banach space and its relation to the approximation problem. Israel J. Math. $13(1972) .30110$.

11 J. Lindenstrauss and L. Trafriri. Classical Banach Spaces 1, Sequerce Spares, Results in Mathematics and Related Areas 92 (Berlin: Springer, 1977).

12 J. S. Morell and J. R. Retherford. p-trivial Banach spaces. Studia Math. 43 (1972), 1-25.

13 A. Pietsch. Operator Ideals (VEB Deutscher Verlag der Wissenschaftens 1978; Amsterdam: NorthHolland, 1980). 
14 G. Pisier. Un théorème sur les opérateurs entre espaces de Banach qui se factorisent par un espace de Hilbert. Ann. Sci. École Norm. Sup. 13 (1980), 23-43.

15 G. Pisier. Counterexample to a conjecture of Grothendicek. Acta Math. 151 (1983), 180-208.

16 C. Samucl. Bounded approximate identities in the algebra of compact operators on a Banach space Proc. Amer. Math. Soc, 117 (1993), 1093-6.

17 Y. V. Selivanov. Homological characterizations of the approximation property for Banach spaces Glasgow Math. J, 34 (1992), 229-39.

18 A. Szankowski. Subspaces without the approximation property. Israel J. Math. 30 [1978\}, 123-9.

19 G. A. Willis. The compact approximation property does not imply the approximation property. Studia Math. 103 (1992), 99-108.

(Issued 16 April 1996)

Downloaded from https:/www.cambridge.org/core. University of Basel Library, on 30 May 2017 at 21:18:28, subject to the Cambridge Core terms of use, available at 